(C) The Authors 2015. This is an Open Access article, distributed under the terms of the Creative Commons Attribution licence (http:// creativecommons.org/licenses/by/3.0/), which permits unrestricted re-use, distribution, and reproduction in any medium, provided the original work is properly cited.

\title{
Probiotic supplementation prevents high-fat, overfeeding-induced insulin resistance in human subjects
}

\author{
Carl J. Hulston $^{1 *}$, Amelia A. Churnside ${ }^{1}$ and Michelle C. Venables ${ }^{2}$ \\ ${ }^{1}$ School of Sport, Exercise and Health Sciences, Loughborough University, Loughborough, Leicestershire LE11 $3 T U$, UK \\ ${ }^{2}$ MRC Human Nutrition Research, Elsie Widdowson Laboratory, Fulbourn Road, Cambridge CB1 9NL, UK
}

(Submitted 30 May 2014 - Final revision received 7 September 2014 - Accepted 6 November 2014 - First published online 29 January 2015)

\begin{abstract}
The purpose of the present study was to determine whether probiotic supplementation (Lactobacillus casei Shirota (LcS)) prevents dietinduced insulin resistance in human subjects. A total of seventeen healthy subjects were randomised to either a probiotic ( $n$ 8) or a control ( $n$ 9) group. The probiotic group consumed a LcS-fermented milk drink twice daily for 4 weeks, whereas the control group received no supplementation. Subjects maintained their normal diet for the first 3 weeks of the study, after which they consumed a high-fat (65\% of energy), high-energy ( $50 \%$ increase in energy intake) diet for $7 \mathrm{~d}$. Whole-body insulin sensitivity was assessed by an oral glucose tolerance test conducted before and after overfeeding. Body mass increased by $0.6($ se $0 \cdot 2) \mathrm{kg}$ in the control group $(P<0 \cdot 05)$ and by $0 \cdot 3(\mathrm{se} 0 \cdot 2) \mathrm{kg}$ in the probiotic group $(P>0 \cdot 05)$. Fasting plasma glucose concentrations increased following $7 \mathrm{~d}$ of overeating (control group: $5 \cdot 3$ (sE $0 \cdot 1$ ) $v .5 .6$ (sE 0.2$) \mathrm{mmol} / 1$ before and after overfeeding, respectively, $P<0.05)$, whereas fasting serum insulin concentrations were maintained in both groups. Glucose AUC values increased by $10 \%$ (from 817 (SE 45 ) to 899 (SE 39) mmol/1 per 120 min, $P<0.05$ ) and whole-body insulin sensitivity decreased by $27 \%$ (from 5.3 (se 1.4 ) to 3.9 (sE 0.9 ), $P<0.05$ ) in the control group, whereas normal insulin sensitivity was maintained in the probiotic group ( 4.4 (SE 0.8$)$ and 4.5 (SE 0.9$)$ before and after overeating, respectively $(P>0.05)$. These results suggest that probiotic supplementation may be useful in the prevention of diet-induced metabolic diseases such as type 2 diabetes
\end{abstract}

Key words: Insulin resistance: High-fat diets: Probiotics

Insulin resistance is a major characteristic of obesity and type 2 diabetes. A number of metabolically active tissues and several potential mechanisms have been implicated in the pathophysiology of insulin resistance, such as impaired GLUT4 translocation to the cell membrane and reduced skeletal muscle glucose uptake ${ }^{(1)}$, elevated hepatic glucose production $^{(2)}$ and impaired $\beta$-cell function, which lead to reduced insulin secretion ${ }^{(3)}$. In addition to these well-described defects, there is emerging evidence to suggest that changes in the gut microbiota might also play an important role in the development of human metabolic disease, through a mechanism that is linked to increased gut permeability, metabolic endotoxaemia and systemic low-grade inflammation ${ }^{(4-7)}$

A clear association has been demonstrated between metabolic disease and compositional changes in the gut microbiota, with a lower abundance of Firmicutes and a higher proportion of Bacteroidetes and Proteobacteria in type 2 diabetic patients when compared with non-diabetic controls ${ }^{(8)}$. Similar results have been found in rodent studies in which insulin resistance is induced via short-term, high-fat, overfeeding ${ }^{(7)}$. In addition to altering the composition of the gut microbiota, high-fat diets have been shown to elevate systemic lipopolysaccharide (LPS) concentrations ${ }^{(9,10)}$, whereas selective modification of the gut microbiota, through prebiotic supplementation, has been shown to reduce high-fat diet-induced metabolic endotoxaemia and lower intestinal permeability in obese diabetic mice ${ }^{(6)}$. Furthermore, probiotic supplementation has been shown to improve glycaemic control and reduce metabolic endotoxaemia in diet-induced obese mice $^{(11)}$. Therefore, prebiotic and/or probiotic supplementation may be a useful strategy to improve metabolic health and prevent diet-induced insulin resistance and type 2 diabetes in humans.

Consumption of probiotic yogurt has already been shown to reduce fasting blood glucose concentrations and glycosylated $\mathrm{Hb}$ levels in type 2 diabetic patients ${ }^{(12)}$, but whether or not probiotics can also prevent diet-induced insulin resistance in otherwise healthy subjects is not yet known. Therefore, we tested the hypothesis that 4 weeks of supplementation with probiotics (Lactobacillus casei Shirota (LCS)) would prevent insulin resistance induced by short-term, high-fat, overfeeding in healthy young males and females. In the present study,

Abbreviations: LCS, Lactobacillus casei Shirota; LPS, lipopolysaccharide; OGTT, oral glucose tolerance test.

*Corresponding author: Dr C. J. Hulston, email c.j.hulston@lboro.ac.uk 
we provide novel evidence that probiotics preserve glycaemic control and prevent insulin resistance during a dietary challenge consisting of severe lipid overload, suggesting that probiotics might be useful in the fight against human metabolic disease.

\section{Methods \\ Subjects}

A total of seventeen healthy subjects (fourteen males and three females; Table 1) volunteered for the present study. They were recruited from the student population and the local community. No payments or other incentives were made for participation. The sample size for the present experiment was estimated based on pilot data from our laboratory in which we observed a large decrease in insulin sensitivity after short-term, high-fat, overfeeding. The inclusion criteria required subjects to be physically active (exercising at least three times per week for more than $30 \mathrm{~min}$ at a time), non-smokers, free from cardiovascular or metabolic disease and not taking any medication, weight stable for at least 6 months, and with a normal BMI $\left(18.5-24.9 \mathrm{~kg} / \mathrm{m}^{2}\right)$. Subjects were excluded from the study if they had taken any probiotic or prebiotic supplements within the previous 3 months. Due to the nature of the dietary intervention, vegetarians and vegans were excluded from the study. The present study was conducted according to the guidelines laid down in the Declaration of Helsinki, and all procedures involving human subjects were approved by the Loughborough University Ethics Committee for Human Participants. Written informed consent was obtained from all subjects.

\section{Pre-testing/pre-screening}

Subjects visited the laboratory 3 weeks before the start of the study for an initial pre-screening, which included an assessment of BMI and fasting plasma glucose and insulin levels.

\section{General study design}

After the initial pre-screening visit, subjects were randomly assigned to one of the following two groups: control group ( $n$ 9, seven males and two females); probiotic group ( $n 8$, seven males and one female). The probiotic that was used in the present study was LcS (commercially available as the fermented milk drink Yakult Light). Both groups maintained their habitual food intake during the first 3 weeks of the study (days 1-21); however, the probiotic group also consumed $65 \mathrm{ml}$ of Yakult Light twice each day. An oral glucose tolerance test (OGTT) was performed on day 22 for the assessment of baseline insulin sensitivity. After completing the first OGTT, subjects were provided with a high-fat (65\% energy), high-energy (approximately 50\% increase in energy intake) diet for $7 \mathrm{~d}$. The probiotic group continued to consume $65 \mathrm{ml}$ of Yakult Light twice throughout the $7 \mathrm{~d}$ overfeeding period. We selected a 4-week LcS supplementation period, as previous experiments have demonstrated that this is sufficient to alter the composition of the gut microbiota in humans ${ }^{(13-15)}$. On day 29, both groups returned to the laboratory for a second OGTT for the assessment of postoverfeeding insulin sensitivity.

\section{Experimental procedures}

On the experimental days (days 22 and 29: OGTT), subjects arrived at the laboratory in the morning (between 07.00 and 09.00 hours) after an overnight fast of at least $10 \mathrm{~h}$. After voiding and being weighed, a twenty-gauge Teflon catheter (Venflon; Becton Dickinson) was inserted into an antecubital vein of one arm to allow repeated blood sampling during the $2 \mathrm{~h}$ OGTT. A fasted blood sample $(10 \mathrm{ml} ; t=0)$ was obtained before subjects ingested a $25 \%$ glucose solution ( $75 \mathrm{~g}$ of glucose dissolved in $300 \mathrm{ml}$ of water). Additional $10 \mathrm{ml}$ blood samples were obtained at 15, 30, 45, 60, 90 and $120 \mathrm{~min}$ after glucose ingestion. Blood samples were divided equally between Vacutainer tubes containing either $\mathrm{K}_{2}$ EDTA or a clotting catalyst (Becton Dickinson) for separation of plasma and serum, respectively. EDTA tubes were stored on ice, whereas serum tubes were left at room temperature until complete clotting had occurred. Blood tubes were then centrifuged at $2300 \mathrm{~g}$ for $10 \mathrm{~min}$ (EDTA, $4^{\circ} \mathrm{C}$; serum $20^{\circ} \mathrm{C}$ ), and the resulting plasma or serum was removed and stored at $-20^{\circ} \mathrm{C}$ until later analysis.

\section{Diet records, analysis and compliance during overfeeding}

Subjects were provided with standardised forms and digital kitchen scales for the purpose of recording weighed food

Table 1. Physical characteristics of the study subjects before and after $7 \mathrm{~d}$ of overeating (Mean values with their standard errors)

\begin{tabular}{|c|c|c|c|c|c|c|c|c|}
\hline & \multicolumn{4}{|c|}{$\begin{array}{c}\text { Control group } \\
\text { ( } n \text { 9; seven males, two females) }\end{array}$} & \multicolumn{4}{|c|}{$\begin{array}{c}\text { Probiotic group } \\
\text { ( } n \text { 8; seven males, one female) }\end{array}$} \\
\hline & \multicolumn{2}{|c|}{ Baseline } & \multicolumn{2}{|c|}{ Overfed } & \multicolumn{2}{|c|}{ Baseline } & \multicolumn{2}{|c|}{ Overfed } \\
\hline & Mean & SE & Mean & SE & Mean & SE & Mean & SE \\
\hline Age (years) & 24 & 2 & - & - & 25 & 2 & - & - \\
\hline Height $(m)$ & 1.72 & 0.02 & - & - & 1.77 & 0.03 & _- & _- \\
\hline Body mass $(\mathrm{kg})$ & $72 \cdot 1$ & $4 \cdot 8$ & $72 \cdot 7^{\star}$ & 4.8 & $73 \cdot 4$ & $2 \cdot 3$ & 73.7 & $2 \cdot 4$ \\
\hline BMI $\left(\mathrm{kg} / \mathrm{m}^{2}\right)$ & $24 \cdot 2$ & 1.2 & $24 \cdot 4^{*}$ & $1 \cdot 2$ & 23.5 & 0.6 & 23.6 & 0.7 \\
\hline
\end{tabular}

* Mean value was significantly different from that at baseline $(P<0.05)$. 
intake on $3 \mathrm{~d}$ each week during the pre-experimental period (days 1-21). Subjects also received detailed written and verbal instructions on how best to complete these records. These records were then used for the assessment of habitual energy intake and diet composition so that individualised diets could be planned and prepared for the $7 \mathrm{~d}$ overfeeding period. The analysis of diet records and planning of food intake for the overfeeding period were performed using WISP version 4.0 (Tinuviel Software). The overfeeding period was designed to increase energy intake by $50 \%$ compared with habitual food intake and consisted of foods that were predominantly high in fat $(65 \%$ of energy consumed as fat). During the overfeeding period, all food was purchased and prepared by the research team and then delivered to the subjects. Subjects were instructed to eat everything that was provided to them, not to eat any other additional foods, and to return any unwanted food so that it could be weighed and the diet values adjusted if necessary. All subjects were informed about the importance of strict diet adherence, and we were confident that the subjects were fully compliant with the diet intervention.

\section{Blood analysis}

Plasma samples were analysed by commercially available spectrophotometric assays for the determination of glucose (Glucose PAP; Horiba Medical) and TAG (Triglyceride PAP; Horiba Medical) concentrations using a semi-automatic analyser (Pentra 400; Horiba Medical). The CV ranged between 0.4 and $0.8 \%$ for plasma samples with high $(10.9 \mathrm{mmol} / 1$, glucose ingestion) and normal $(4.7 \mathrm{mmol} / \mathrm{l}$, fasted) glucose concentrations. The CV ranged between 1.6 and $3.4 \%$ for plasma samples with normal $(1.1 \mathrm{mmol} / \mathrm{l})$ and low $(0.5 \mathrm{mmol} / \mathrm{l})$ TAG concentrations.

Serum insulin concentrations were analysed by an enzymelinked immunosorbent assay (ELISA EIA-2935; DRG Instruments $\mathrm{GmbH}$ ). The $\mathrm{CV}$ for serum insulin analysis was 3.0\% for a mid-range sample (375 pmol/1, glucose ingestion).

\section{Calculations}

Plasma glucose and serum insulin concentrations obtained from the OGTT were used to assess whole-body insulin sensitivity using the Matsuda insulin sensitivity index (ISI):

$$
\mathrm{ISI}=\frac{10000}{\sqrt{(\text { FPG } \times \text { FPI }) \times(\text { mean OGTT insulin concentration })}}
$$

where FPG is the fasting plasma glucose concentration; FPI is the fasting plasma insulin concentration; and 10000 represents a constant that allows numbers ranging between 1 and 12. The square root conversion is used to correct the non-linear distribution of values ${ }^{(16)}$.

\section{$A \cup C$}

The AUC was calculated using the trapezoidal rule with zero as the baseline.

\section{Statistical analysis}

The primary outcome measurement was a change in insulin sensitivity (determined from plasma glucose and serum insulin concentrations during an OGTT). Secondary outcome measurements were changes in body mass, BMI and fasting plasma TAG concentrations. Data analysis was performed using SPSS version 21.0 for Windows (SPSS, Inc.). Data are presented as means with their standard errors. To compare potential differences in metabolic responses to overfeeding, a two-way (pre- $v$. post-overfeeding) repeated-measures ANOVA was conducted with a between-subject variable (control $v$. probiotic group) followed by post hoc analysis where appropriate. The level of significance was set at $P<0.05$.

\section{Results}

\section{Weight gain and $B M /$ with overeating}

By the end of the $7 \mathrm{~d}$ overfeeding period, subjects in the control group had gained a body mass of 0.6 ( $\operatorname{se~} 0.2) \mathrm{kg}(P<0.05)$, whereas the increase in body mass for those in the probiotic group was smaller and not significant (0.3 (SE 0.2$) \mathrm{kg}$, $P>0.05)$. No significant changes in BMI occurred as a result of $7 \mathrm{~d}$ of overeating. Data for body mass and BMI are summarised in Table 1.

\section{Fasting plasma substrates and serum insulin}

Fasting plasma glucose concentrations increased following $7 \mathrm{~d}$ of overeating (control group: $5 \cdot 3$ (SE 0.1) v. $5 \cdot 6$ (SE 0.2) mmol/1 before and after overfeeding, respectively, $P<0 \cdot 05)$, whereas fasting serum insulin concentrations were well maintained in both groups. Fasting plasma TAG concentrations were decreased following overeating (both groups, $P<0 \cdot 05$ ). Fasting data summarised in Table 2 were obtained from the zero time point (before glucose ingestion) of the OGTT that was performed before and after overfeeding.

\section{Energy intake and diet composition}

Habitual energy intake and diet composition were similar between the control and probiotic groups (Table 3). The intentional overfeeding period increased energy intake by 51.9 and $51.3 \%$ in the control and probiotic groups, respectively $(P<0.05)$. The majority of this additional energy was provided by an increase in dietary fat intake.

\section{Oral glucose tolerance test}

The plasma glucose and serum insulin responses to an OGTT before and after $7 \mathrm{~d}$ of overfeeding are shown in Fig. 1 . In the control group, overfeeding caused a $10 \%$ increase in glucose AUC values (from 817 (SE 45) to 899 (SE 39) mmol/l per 120 min, $P<0.05$; Fig. 1(a)), whereas no change was observed in the probiotic group (baseline: 866 ( $\mathrm{SE} 49$ ) $\mathrm{mmol} / \mathrm{l}$ per $120 \mathrm{~min}$; overfed: 888 (SE 53) mmol/1 per $120 \mathrm{~min}$; Fig. 1(b)). A similar trend was observed for insulin responses (Fig. 1(c) and (d)), but did not reach significance. 
Table 2. Fasting plasma substrate and serum insulin concentrations before and after $7 \mathrm{~d}$ of overeating

(Mean values with their standard errors)

\begin{tabular}{|c|c|c|c|c|c|c|c|c|}
\hline & \multicolumn{4}{|c|}{$\begin{array}{c}\text { Control group } \\
\text { ( } n \text { 9; seven males, two females) }\end{array}$} & \multicolumn{4}{|c|}{$\begin{array}{c}\text { Probiotic group } \\
\text { ( } n \text { 8; seven males, one female) }\end{array}$} \\
\hline & \multicolumn{2}{|c|}{ Baseline } & \multicolumn{2}{|c|}{ Overfed } & \multicolumn{2}{|c|}{ Baseline } & \multicolumn{2}{|c|}{ Overfed } \\
\hline & Mean & SE & Mean & SE & Mean & SE & Mean & SE \\
\hline Glucose $(\mathrm{mmol} / \mathrm{l})$ & $5 \cdot 3$ & 0.1 & $5 \cdot 6^{\star}$ & 0.2 & $5 \cdot 8$ & 0.1 & $5 \cdot 8$ & 0.1 \\
\hline Insulin (pmol/l) & 86 & 9 & 86 & 11 & 81 & 9 & 75 & 12 \\
\hline TAG $(\mathrm{mmol} / \mathrm{l})$ & 1.4 & 0.3 & $0.9^{*}$ & 0.1 & 1.4 & 0.2 & $1.0^{*}$ & 0.2 \\
\hline
\end{tabular}

${ }^{\star}$ Mean value was significantly different from that at baseline $(P<0.05)$.

\section{Insulin sensitivity}

After $7 \mathrm{~d}$ of overfeeding, whole-body insulin sensitivity in the control group was impaired by $27 \%$ (decreased from 5.3 (SE 1.4) to 3.9 (SE 0.9), $P<0.05$ ), whereas no change in insulin sensitivity was observed in the probiotic group (Fig. 2).

\section{Discussion}

The main finding of the present study was that high-fat overfeeding decreased insulin sensitivity by approximately $27 \%$ in healthy young males and females; however, supplementation with the LcS probiotic before and throughout the overfeeding period preserved glycaemic control and maintained insulin action. These results provide further indirect evidence that compositional changes in the gut microbiota lead to the development of human metabolic disease and that probiotic supplementation could be useful in preventing insulin resistance induced by excessive consumption of high-fat foods (i.e. a Westernised diet).

Numerous rodent studies have reported that short-term (3 d to 4 weeks) adherence to a high-fat diet induces insulin resistance $^{(17-23)}$, and similar results have been reported in a small number of recent human studies ${ }^{(2,24,25)}$, because of which we chose to adopt this model in the present study. Although the underlying mechanisms for the development of insulin resistance remain unclear, a popular theory is that excessive consumption of high-fat foods can lead to the accumulation of reactive intra-myocellular lipids, such as ceramide and diacylglycerol, which inhibit insulin signalling (insulin/IRS-1/
PI3-K/AKT pathway) and impair GLUT4 translocation to the cell membrane, thereby reducing skeletal muscle glucose uptake $^{(26-28)}$. Another potential mechanism is systemic lowgrade inflammation, as the prevalence of insulin resistance is associated with an increase in LPS (a component of the cell wall of Gram-negative bacteria) concentrations in the blood $^{(29,30)}$. Furthermore, in experimental models of metabolic endotoxaemia, using intravenous or subcutaneous LPS infusion, it has been found that LPS acts as a trigger for proinflammatory cytokine production ${ }^{(4,31)}$, which, in turn, leads to impaired insulin action through increased serine phosphorylation of insulin receptor substrate- $1^{(31,32)}$. Interestingly, the increase in systemic LPS levels that is associated with the metabolic syndrome is thought to originate from the Gramnegative bacteria living in the gut ${ }^{(5-7,10,30,33)}$. Normally, the intestinal epithelium would act as a physical barrier to prevent LPS translocation into the circulation. However, reduced expression of intestinal epithelial tight junction proteins ( $\mathrm{ZO}-1$ and occludin) and increased gut permeability (i.e. decreased gut barrier function) have been observed in animal models of insulin resistance and type 2 diabetes ${ }^{(5,6)}$. This decrease in gut barrier function may be causally linked to diet-induced changes in the composition of the gut microbiota, as highfat diets have been shown to increase the ratio of Bacteroidetes (Gram-negative) to Firmicutes (Gram-positive) and increase gut permeability that led to the development of diabetes in mice ${ }^{(7)}$. Furthermore, selectively increasing the abundance of Bifidobacterium spp., through prebiotic supplementation, has been shown to reduce intestinal permeability in obese diabetic mice ${ }^{(6)}$. In the context of the

Table 3. Daily energy intake and diet composition before and after $7 d$ of overeating (Mean values with their standard errors)

\begin{tabular}{|c|c|c|c|c|c|c|c|c|}
\hline & \multicolumn{4}{|c|}{$\begin{array}{c}\text { Control group } \\
\text { ( } n \text { 9; seven males, two females) }\end{array}$} & \multicolumn{4}{|c|}{$\begin{array}{c}\text { Probiotic group } \\
\text { ( } n \text { 8; seven males, one female) }\end{array}$} \\
\hline & \multicolumn{2}{|c|}{ Baseline } & \multicolumn{2}{|c|}{ Overfed } & \multicolumn{2}{|c|}{ Baseline } & \multicolumn{2}{|c|}{ Overfed } \\
\hline & Mean & SE & Mean & SE & Mean & SE & Mean & SE \\
\hline Energy (kJ) & 9934 & 759 & $15091^{*}$ & 1079 & 10751 & 581 & $16265^{*}$ & 865 \\
\hline Protein $(\mathrm{g})$ & 97 & 8 & $117^{*}$ & 9 & 103 & 6 & $127^{*}$ & 8 \\
\hline Carbohydrate (g) & 292 & 17 & $213^{\star}$ & 13 & 317 & 11 & $226^{\star}$ & 15 \\
\hline Fat $(\mathrm{g})$ & 91 & 11 & $259^{*}$ & 11 & 103 & 12 & $281^{*}$ & 15 \\
\hline
\end{tabular}

${ }^{*}$ Mean value was significantly different from that at baseline $(P<0.05)$. 

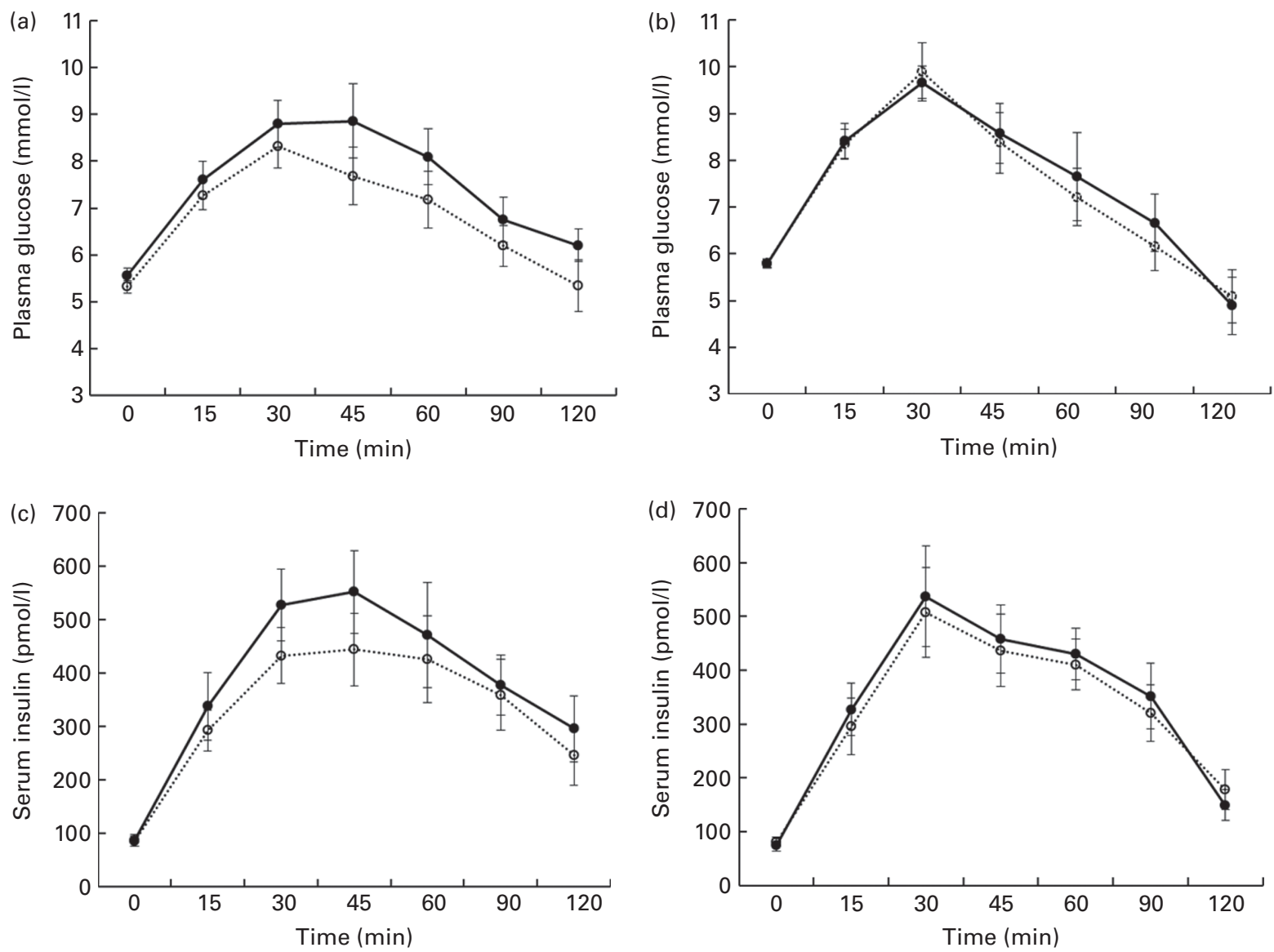

Fig. 1. Plasma glucose ((a) control group and (b) probiotic group) and serum insulin ((c) control group and (d) probiotic group) concentrations during a $2 \mathrm{~h}$ oral glucose tolerance test conducted before and after $7 \mathrm{~d}$ of overeating. Values are means, with their standard errors represented by vertical bars ( $n 9$, control group; $n 8$ probiotic group). $\odot$, Baseline; - -, overfed.

present study, this tends to suggest that the ability of probiotic supplementation to prevent high-fat diet-induced insulin resistance is most probably due to the maintenance of a favourable gut microbiota and preservation of gut barrier function, although further mechanistic work will be necessary to confirm this finding.

The main limitation of the present study was that we cannot confirm whether the high-fat diet altered the composition of the gut microbiota, impaired gut barrier function or led to an increase in systemic inflammation, nor can we confirm the impact of LcS probiotic supplementation on these physiological outcomes. Although it was not possible to identify the underlying mechanisms in the present study, we have discussed our observations and speculated upon the possible causes in the context of what has already been shown in animals. It is also important to keep in mind that this was a preliminary, proof-of-concept investigation into the efficacy for probiotic supplementation to maintain human metabolic health. Therefore, it was important to assess the changes in whole-body insulin sensitivity (i.e. the primary outcome measurement), and to confirm our experimental hypothesis, before conducting the more costly and time-consuming mechanistic experiments. In future studies, it will be necessary to perform faecal DNA analysis in order to determine diet- and/or probiotic-induced changes in gut microbiota composition, as well as to measure the markers of gut barrier function and changes in systemic and tissue inflammation. Nonetheless, the preliminary finding that 4 weeks of LcS probiotic supplementation maintained glycaemic control and

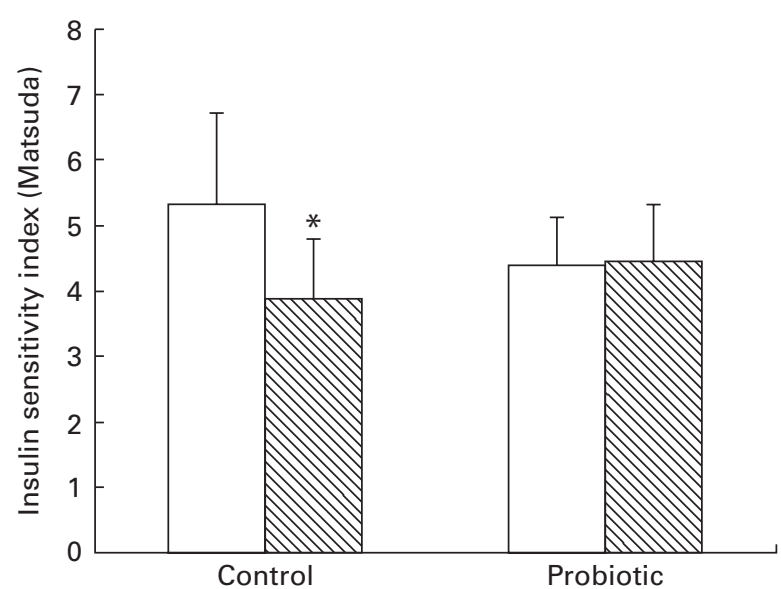

Fig. 2. Matsuda insulin sensitivity index calculated during an oral glucose tolerance test conducted before and after $7 \mathrm{~d}$ of overeating for the control and probiotic groups. Values are means, with their standard errors represented by vertical bars ( $n 9$, control group; $n 8$ probiotic group). * Mean value was significantly different from that at baseline $(P<0.05) . \square$, Baseline;

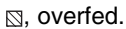


prevented insulin resistance during a dietary challenge that consisted of severe lipid overload (approximately $270 \mathrm{~g}$ of fat/d) suggests that diet-induced changes in the gut microbiota are likely to play an important role in human metabolic health and disease. Anti-diabetic properties of certain probiotics have been reported in several rodent studies ${ }^{(1,34-37)}$ and in one human study ${ }^{(12)}$. In the latter study, sixty-four type 2 diabetic patients consumed either a probiotic yogurt containing Lactobacillus acidophilus La5 and Bifidobacterium lactis Bb12 or a placebo, probiotic-free, yogurt for 6 weeks. The authors have reported a significant decrease in both fasting blood glucose and glycosylated $\mathrm{Hb}$ concentrations as well as an improvement in antioxidant status. We did not find a reduction in fasting glucose or insulin concentrations following probiotic supplementation, most probably because of the fact that we tested healthy young subjects with normal baseline values. However, the present results demonstrate that regular consumption of LcS probiotics may offer protection against the development of diet-induced insulin resistance. Not all studies have reported metabolic health benefits following LcS supplementation. Triplot et al. ${ }^{(38)}$ reported that 12 weeks of supplementation with LcS failed to improve insulin sensitivity or enhance $\beta$-cell function in subjects with the metabolic syndrome. The discrepancy between their previous findings and the present study may be related to the recruitment of a clinical population as opposed to healthy volunteers. With this in mind, it is reasonable to suggest that LCS probiotics could be used more effectively in the prevention, rather than in the treatment, of human metabolic disease.

We observed a significant decrease in fasting plasma TAG concentrations after $7 \mathrm{~d}$ of overfeeding, which is consistent with the findings of another very recent study ${ }^{(39)}$. However, the reduction in fasting plasma TAG concentrations is most probably due to changes in the macronutrient composition of the diet, rather than the amount of energy consumed. Removing carbohydrate from the diet and replacing it with an isoenergetic amount of fat has been shown to reduce plasma TAG concentrations ${ }^{(40)}$, whereas increasing the amount of carbohydrate in the diet has been shown to have the opposite effect and actually increases fasting plasma TAG concentrations ${ }^{(41)}$. This effect has been termed carbohydrate-induced hypertriacylglycerolaemia ${ }^{(42)}$. In the present study, the amount of carbohydrate consumed during the overfeeding period was reduced in both absolute and relative terms when compared with the subject's habitual food intake (approximately 220 v. $305 \mathrm{~g} / \mathrm{d}$ ), and additional energy intake was met through a large increase in the amount of fat that was consumed, suggesting that the reduction in fasting plasma TAG concentration may be related to the removal of carbohydrate from the diet. Without the use of stable isotope tracers, it is difficult to speculate on the reason for a reduction in plasma TAG concentration after overfeeding; however, it must be related to changes in TAG turnover: be it a decrease in the synthesis rate, an increase in the clearance rate, or a combination of both.

In conclusion, we have shown that probiotic supplementation has the potential to prevent high-fat diet-induced insulin resistance in human subjects, and this warrants confirmation in a larger, placebo-controlled study. Further mechanistic studies are also necessary to confirm the underlying mechanism for this effect.

\section{Acknowledgements}

The present study was conceptualised and initiated by the lead investigator (C. J. H.), and was financially supported by industry funds. The cost of consumables for the study was covered by an educational grant from Yakult UK Limited. Yakult UK Limited had no role in the design and analysis of the study or in the writing of this article. A. A. C. was supported by a summer studentship grant from the Society for Endocrinology. M. C. V. was funded by the Medical Research Council (grant no. U1059·60·389).

The authors' contributions are as follows: C. J. H. designed the study, collected the data and wrote the manuscript; A. A. C. collected the data and assisted with the preparation of the manuscript; M. C. V. assisted with the sample analysis and contributed to the preparation of the manuscript.

The authors declare that there are no conflicts of interest.

\section{References}

1. Tremblay F, Lavigne C, Jacques H, et al. (2001) Defective insulin-induced GLUT4 translocation in skeletal muscle of high fat-fed rats is associated with alterations in both Akt/ protein kinase $\mathrm{B}$ and atypical protein kinase $\mathrm{C}$ activities. Diabetes 50, 1901-1910.

2. Brons C, Jensen CB, Storgaard H, et al. (2009) Impact of short-term high-fat feeding on glucose and insulin metabolism in young healthy men. $J$ Physiol 587, 2287-2297.

3. Defronzso RA (2004) Dysfunctional fat cells, lipotoxicity and type 2 diabetes. Int J Clin Pract 58, 9-21.

4. Cani PD, Amar J, Iglesias MA, et al. (2007) Metabolic endotoxemia initiates obesity and insulin resistance. Diabetes 56, 1761-1772.

5. Cani PD, Bibiloni R, Knauf C, et al. (2008) Changes in gut microbiota control metabolic endotoxemia-induced inflammation in high-fat diet-induced obesity and diabetes in mice. Diabetes 57, 1470-1481.

6. Cani PD, Possemiers S, van de Wiele T, et al. (2009) Changes in gut microbiota control inflammation in obese mice through a mechanism involving GLP-2-driven improvement of gut permeability. Gut 58, 1091-1103.

7. Serino M, Luche E, Gres S, et al. (2012) Metabolic adaptations to a high-fat diet is associated with a change in the gut microbiota. Gut 61, 543-553.

8. Larsen N, Vogensen FK, van den Berg FWJ, et al. (2010) Gut microbiota in human adults with type 2 diabetes differs from non-diabetic adults. PloS ONE 5, e9085.

9. Amar J, Burcelin R, Ruidavets JB, et al. (2008) Energy intake is associated with endotoxemia in apparently healthy men. Am J Clin Nutr 87, 1219-1223.

10. Pendyala S, Walker JM \& Holt PR (2012) A high-fat diet is associated with endotoxemia that originates in the gut. Gastroenterology 142, 1100-1101.

11. Naito E, Yoshida Y, Makino K, et al. (2011) Beneficial effect of oral administration of Lactobacillus case i strain Shirota on insulin resistance in diet-induced obesity mice. J App Microbiol 110, 650-657. 
12. Ejtahed HS, Mohtadi-Nia J, Homayouni-Rad A, et al. (2012) Probiotic yogurt improves antioxidant status in type 2 diabetic patients. Nutrition 28, 539-543.

13. Spanhaak S, Havenaar R \& Schaafsma G (1998) The effect of consumption of milk fermented by Lactobacillus casei strain Shirota on the intestinal microflora and immune parameters in humans. Eur J Clin Nutr 52, 899-907.

14. Matsumoto K, Takada T, Shimizu K, et al. (2010) Effects of a probiotic fermented milk beverage containing Lactobacillus casei strain Shirota on defecation frequency, intestinal microbiota, and the intestinal environment of healthy individuals with soft stools. J Biosci Bioeng 110, 547-552.

15. Nagata S, Asahara T, Ohta T, et al. (2011) Effect of the continuous intake of probiotic-fermented milk containing Lactobacillus casei strain Shirota on fever in a mass outbreak of norovirus gastroenteritis and the faecal microflora in a health service facility for the aged. Br J Nutr 106, 549-556.

16. Matsuda M \& DeFronzo RA (1999) Insulin sensitivity indices obtained from oral glucose tolerance testing: comparison with the euglycemic insulin clamp. Diabetes Care 22, 1462-1470.

17. Kraegen EW, Storlien LH, Jenkins AB, et al. (1989) Chronic exercise compensates for insulin resistance induced by a high-fat diet in rats. Am J Physiol Endocrinol Metab 256, E242-E249.

18. Kim C, Youg JH, Park J, et al. (2000) Effects of high-fat diet and exercise training on intracellular glucose metabolism in rats. Am J Physiol Endocrinol Metab 278, E977-E984.

19. Lessard SJ, Rivas DA, Chen K, et al. (2007) Tissue-specific effects of rosiglitazone and exercise in the treatment of lipid-induced insulin resistance. Diabetes 56, 1856-1864.

20. Todd MK, Watt MJ, Le J, et al. (2007) Thiazolidinediones enhanced skeletal muscle triacylglycerol synthesis while protecting against fatty acid-induced inflammation and insulin resistance. Am J Physiol Endocrinol Metab 292, E485-E493.

21. Barclay JL, Shostak A, Leliavski A, et al. (2013) High fat diet-induced hyperinsulinemia and tissue-specific insulin resistance in Cry deficient mice. Am J Physiol Endocrinol Metab 304, E1053-E1063.

22. Bielohuby M, Sisley S, Sandoval D, et al. (2013) Impaired glucose tolerance in rats fed low-carbohydrate, high-fat diets. Am J Physiol Endocrinol Metab 305, E1059-E1070.

23. Wiedemann MSF, Wueest S, Item F, et al. (2013) Adipose tissue inflammation contributes to short-term high-fat dietinduced hepatic insulin resistance. Am J Physiol Endocrinol Metab 305, E388-E395.

24. Adochio RL, Leitner JW, Gray K, et al. (2009) Early responses of insulin signalling to high-carbohydrate and high-fat overfeeding. Nutr Metab 6, 37 .

25. Cornford AS, Hinko A, Nelson RK, et al. (2013) Rapid development of systemic insulin resistance with overeating is not accompanied by robust changes in skeletal muscle glucose and lipid metabolism. Appl Physiol Nutr Metab 38, 512-519.

26. Yu C, Chen Y, Zong H, et al. (2002) Mechanism by which fatty acids inhibit insulin activation of insulin receptor substrate-1 (IRS-1)-associated phosphatidylinositol 3-kinase activity in muscle. J Biol Chem 277, 50230-50236.
27. Kleemann R, van Erk M, Verschuren L, et al. (2010) Timeresolved and tissue-specific systems analysis of the pathogenesis of insulin resistance. PloS ONE 5, e8817.

28. Samuel VT \& Shulman GI (2012) Mechanisms for insulin resistance: common threads and missing links. Cell 148, 852-871.

29. Pussinen PJ, Havulinna AS, Lehto M, et al. (2011) Endotoxemia is associated with an increased risk of incident diabetes. Diabetes Care 34, 392-397.

30. Harte AL, Varma MC, Tripathi G, et al. (2012) High fat intake leads to acute postprandial exposure to circulating endotoxin in type 2 diabetic subjects. Diabetes Care 35, 375-382.

31. Mehta NN, McGillicuddy FC, Anderson PD, et al. (2010) Experimental endotoxemia induces adipose inflammation and insulin resistance in humans. Diabetes 59, 172-181.

32. Hotamisligil G, Peraldi P, Budavari A, et al. (1996) IRS1-mediated inhibition of insulin receptor tyrosine kinase activity in TNF- $\alpha$ and obesity-induced insulin resistance. Science 271, 665-668.

33. Brun P, Castagliuolo I, Di Leo V, et al. (2007) Increased intestinal permeability in obese mice: new evidence in the pathogenesis of nonalcoholic steatohepatitis. Am J Physiol Gastrointest Liver Physiol 292, G518-G525.

34. Matsuzaki T, Yamazaki R, Hashimoto S, et al. (1997) Antidiabetic effects of an oral administration of Lactobacillus case $i$ in a non-insulin-dependent diabetes mellitus (NIDDM) model using KK-Ay mice. Endocr J 44, 357-365.

35. Matsuzaki T, Nagata Y, Kado S, et al. (1997) Prevention of onset in an insulin-dependent diabetes mellitus model, NOD mice, by oral feeding of Lactobacillus casei. APMIS 105, 643-649.

36. Harisa GI, Taha EI, Khalil AF, et al. (2009) Oral administration of Lactobacillus acidophilus restores nitric oxide level in diabetic rats. Aust J Basic Appl Sci 3, 2963-2969.

37. Tanida M, Imanishi K, Akashi H, et al. (2014) Injection of Lactobacillus casei strain Shirota affects autonomic nerve activities in a tissue-specific manner, and regulates glucose and lipid metabolism in rats. J Diabetes Investig 5, 153-161.

38. Triplot NJ, Leber B, Blattl D, et al. (2012) Effect of supplementation with Lactobacillus casei Shirota on insulin sensitivity, $\beta$-cell function, and markers of endothelial function and inflammation in subjects with the metabolic syndrome - a pilot study. J Dairy Sci 96, 89-95.

39. Wulan SN, Westerterp KR \& Plasqui G (2014) Metabolic profile before and after short-term overfeeding with a high-fat diet: a comparison between South Asian and white men. Br J Nutr 111, 1853-1861.

40. Hudgins LC, Hellerstein M, Seidman C, et al. (1996) Human fatty acid synthesis is stimulated by a eucaloric low fat, high carbohydrate diet. J Clin Invest 97, 2081-2091.

41. Retzlaff BM, Walden CE, Dowdy AA, et al. (1995) Changes in plasma triacylglycerol concentrations among free-living hyperlipidemic men adopting different carbohydrate intakes over 2 years: the Dietary Alternatives Study. Am J Clin Nutr 62, $988-995$.

42. Hellerstein MK (2002) Carbohydrate-induced hypertriglyceridemia: modifying factors and implications for cardiovascular risk. Curr Opin Lipidol 13, 33-40. 